\title{
A Photovoltaic Array Fault Diagnosis Method Considering the Photovoltaic Output Deviation Characteristics
}

\author{
Jian Zhao, ${ }^{1}$ Qian Sun, ${ }^{1}$ Ning Zhou, ${ }^{1}$ Hao Liu, ${ }^{1}$ and Haizheng Wang $\mathbb{D}^{2}$ \\ ${ }^{1}$ State Grid Henan Electric Power Company Research Institute, Zhengzhou 450000, China \\ ${ }^{2}$ School of Renewable Energy, North China Electric Power University, Beijing 102206, China \\ Correspondence should be addressed to Haizheng Wang; wanghaizheng@ncepu.edu.cn
}

Received 6 September 2019; Revised 15 January 2020; Accepted 10 February 2020; Published 1 March 2020

Academic Editor: Francesco Riganti-Fulginei

Copyright ( 2020 Jian Zhao et al. This is an open access article distributed under the Creative Commons Attribution License, which permits unrestricted use, distribution, and reproduction in any medium, provided the original work is properly cited.

\begin{abstract}
There are a large number of photovoltaic (PV) arrays in large-scale PV power plants or regional distributed PV power plants, and the output of different arrays fluctuates with the external conditions. The deviation and evolution information of the array output are easily covered by the random fluctuations of the PV output, which makes the fault diagnosis of PV arrays difficult. In this paper, a fault diagnosis method based on the deviation characteristics of the PV array output is proposed. Based on the current of the PV array on the DC (direct current) side, the deviation characteristics of the PV array output under different arrays and time series are analyzed. Then, the deviation function is constructed to evaluate the output deviation of the PV array. Finally, the fault diagnosis of a PV array is realized by using the probabilistic neural network (PNN), and the effectiveness of the proposed method is verified. The main contributions of this paper are to propose the deviation function that can extract the fault characteristics of PV array and the fault diagnosis method just using the array current which can be easily applied in the PV plant.
\end{abstract}

\section{Introduction}

In recent years, the PV industry has developed rapidly as the cost of PV modules has been greatly reduced. The installed capacity of PV power plants is increasing rapidly [1]; by the end of 2018, the cumulative PV installed capacity of China reached $174.63 \mathrm{GW}$, with an additional installed capacity of 44.1 GW. The PV power plant has a large number of modules, which works under the natural environment, so the module or array often failures in PV power plants [2]. A failure in module can degrade the operating efficiency of the PV array and even seriously endanger the safe operation of the PV plant [3]. Therefore, the real-time monitoring of the operating status and timely detection of the PV arrays faults are very important for its effectively operating.

There are two main types of fault diagnosis strategies for DC side in PV plant $[4,5]$. The first type methods rely on the test equipment for PV modules/arrays. And in references $[6,7]$, infrared cameras are used to detect the temperature differences among modules and then identify the fault modules. Madeti et al. [8] diagnosed faults directly by plac- ing sensors on the PV array. Yihua et al. [9] collected voltage data by installing voltage sensors in the arrays and then used these data to realize PV fault diagnosis. Livera et al. [10] summarize the disadvantages of infrared-based fault diagnosis methods and the advantages of PV electrical parameters-based methods, such methods require a large number of test equipment, which greatly increases the cost of diagnosis, so it is difficult to be applied in actual PV power plants. So more and more scholars are trying to use the operational data to develop the fault diagnosis methods of PV plant.

The second type method is based on the operational data from PV array, and such method can be divided into three categories. The first category methods are based on the reference model. Chine et al. [11] used the ANN (artificial neural network) to build the reference model of the PV module. Fouzi et al. [12] and Yang et al. [13] developed the reference model of the PV module from the historical data and used the deviation between the actual and theoretical output for fault diagnosis. Chaibi et al. [14] used an artificial colony optimization algorithm to build the PV model, based on the 
deviation of measured and reference value for fault diagnosis. Through the PV model, Fu et al. [15] and Liu et al. [16] introduced the indicator of the array current dispersion rate of a combiner box. Such type of method can effectively determine the fault type through the deviation analysis, but due to the complex modelling process, the performance differences between modules, and the nonlinear distortion of the PV module output parameters caused by the aging of the PV power plant, the model accuracy is difficult to meet the fault diagnosis requirements.

The second category methods are based on the statistical analysis of running data from PV plant. Mahmoud et al. [17] introduced the PV output indicators and obtained the threshold of indicators by using statistical $t$-test; finally, the threshold is used for fault diagnosis. Based on the statistical analysis of measured and reference value, Majdi et al. [18] proposed a multiscale weighted generalized likelihood ratio test chart for PV fault diagnosis. Garoudjaa et al. [19] combined the residual error between the actual and reference values with the exponentially weighted moving average control chart for fault diagnosis. In reference [20] based on variation between measured and estimated power, a statistical approach was introduced to set thresholds that can be used for locating defects in the PV system. This kind of fault diagnosis method needs to master the prior knowledge of the distribution characteristics of the analyzed objects, but the prior knowledge is difficult to be obtained in advance.

The third category methods are the intelligent classification-based methods. Chen et al. [21] used a principal component analysis and support vector machine to classify the faults in PV systems. Some scholars used the extreme learning machine [22] and fuzzy clustering method [23] to classify the obtained data and then identified the various faults of the PV array. Chen et al. [24] used the random forest ensemble learning algorithm for fault detection of PV array. In reference $[25,26]$, the newly deep residual network model trained by the adaptive moment estimation deep learning algorithm is built for fault diagnosis of PV arrays. The intelligent classification method avoids the complex process of modelling and the classification process is easy to implement, but this method requires a large amount of fault sample data to train the model. Akram and Lotfifard [27] selected the PNN algorithm for PV fault diagnosis by comparing various fault diagnosis methods of PV system. PNN algorithm has good nonlinear learning ability and is suitable for small sample size training, which are the important reasons for choosing PNN to classify the sample in this paper.

Through the above analysis, the methods using operational data of PV array are the most potential fault diagnosis method. But the operational data of the PV array will change with the external environment $[28,29]$, and the output characteristics of the array are easily covered by a large number of data. The author of this paper studied the spatial-temporal distribution characteristics of PV array under different faults [30] and the statistical characteristics of PV array output under different conditions [31]. Based on the above research, designing a classification method for PV fault characteristics are the key to improve the quality of PV fault diagnosis. This paper focuses on the fault feature extraction of PV array output and combines the fault feature extraction method with PNN classification algorithm for fault diagnosis. In the PV power plant, the PV arrays are connected to the combiner box in parallel, and the PV array voltage in one combiner box is the same which is difficult to be used for fault diagnosis of PV array. The main contribution of the paper is to build a new fault diagnosis method of PV array by analyzing the deviation characteristics of different arrays.

In this work, the distribution characteristics of PV array output deviation are studied. And a deviation function that can effectively extract the deviation information of the PV array current is constructed. The rest of the paper is as follows. Section 2 studies the deviation characteristics of PV arrays output currents in PV power plant. In Section 3, the deviation function is established to describe the output deviation of PV array. Section 4 proposes a fault diagnosis method for PV arrays. In Section 5, the experimental verification of the proposed method is carried out. Finally, Section 6 summarizes the major innovation points of this work.

\section{Deviation Characteristics of PV Arrays}

2.1. Output Characteristics of PV Arrays. This paper uses a large-scale PV power plant in China as the object for analysis. This PV power plant consists of 553 intelligent PV combiner boxes and 74 inverters. It has approximately 130,000 PV modules and more than 8000 arrays; each array consists of 16 modules. 16 arrays are connected in parallel in each combiner boxes and 7 combiner boxes are connected to one inverter. The analyzed data in this paper are all from this plant, and the time resolution of the data is 10 minutes. For actual PV power plants, the array current is the main available data for fault diagnosis. Therefore, this paper takes the array current as the analyzed variable.

In order to analyze the output characteristics of different arrays, five arrays with arrays 1-3 are connected in parallel in the same combiner box and the other two arrays are connected in different combiner boxes are selected. Figure 1 shows the current distributions of the five arrays in the PV power plant in 7 consecutive days. The output of each array is similar under normal operation, and it fluctuates wildly as the weather changes. Large-scale PV power plants have a large number of arrays, and the data collected are very complicated. Therefore, to diagnose the fault of PV arrays, the fault characteristics of the PV array must be extracted under complex operating conditions.

2.2. Deviation Characteristics of PV Array Current. To show the deviation characteristics of the PV array output directly, the reference current is introduced to compare with the actual value. The reference current is the theoretical maximum power point current of the PV array, and the calculation formula of the maximum power point current, $I_{m}$, of the PV array is

$$
I_{m}=I_{m-\text { ref }}\left[1+\alpha\left(T-T_{\text {ref }}\right)\right] \frac{G}{G_{\text {ref }}} .
$$




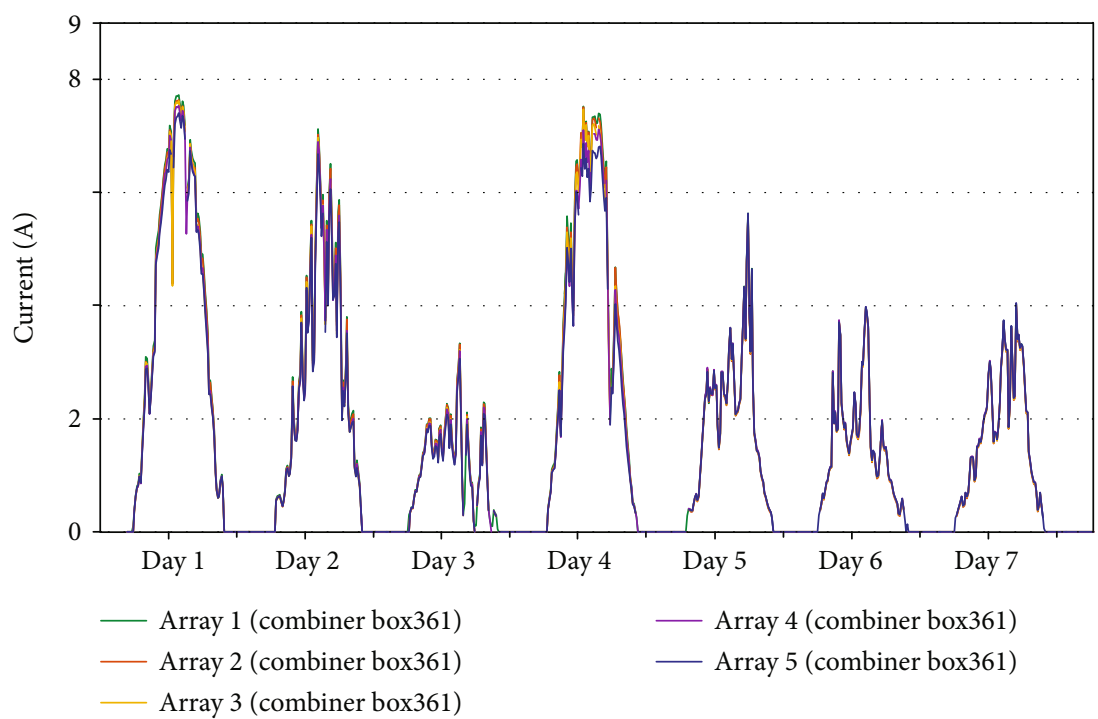

Figure 1: Current distribution of different arrays.

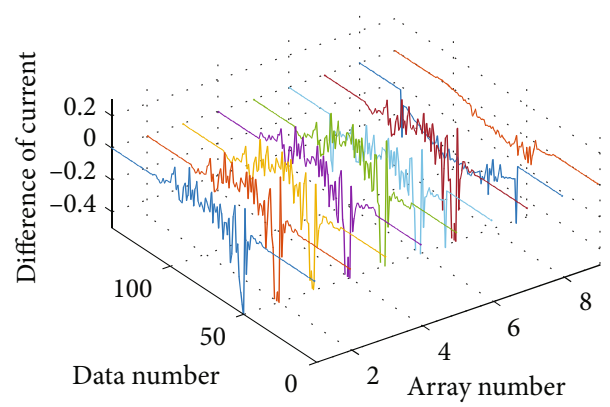

(a)

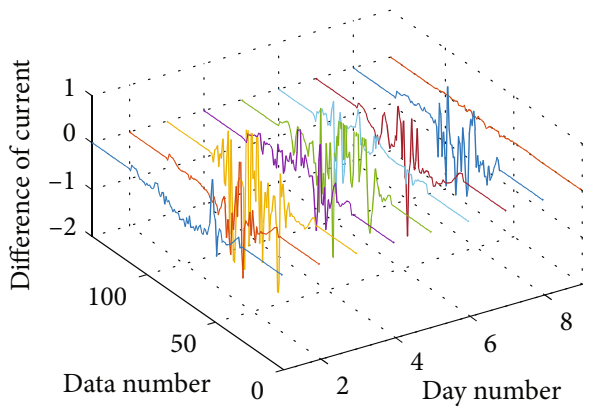

(b)

FiguRE 2: Distribution characteristics of the difference between the array current and the reference current. (a) Different arrays. (b) Different days.

$I_{m-\text { ref }}$ is the maximum power point current of the PV modules in standard test conditions and $\alpha$ is the compensation coefficient. The PV module temperature $T$ is calculated by the ambient temperature [32], $T_{\text {ref }}=25^{\circ} \mathrm{C}$ is the reference temperature, $G$ is the measured solar radiation intensity, which is sampled by the solar pyranometer set in the power plant, and $G_{\text {ref }}=1000 \mathrm{~W} / \mathrm{m}^{2}$.

The current are from nine arrays in the power plant, and Figure 2 shows the distribution of the difference between the array current $I_{T, S}$ and reference current $I_{m}$ of 9 consecutive days, and the 9 days are selected from July $2^{\text {nd }}$ to $10^{\text {th }}$. The sampling interval here is ten minutes, and 138 data points were collected from 0 o'clock to 23 o'clock every day. The difference of current is obtained by subtracting the reference current calculated by Equation (1) from the measured current. Figure 2(a) compares different arrays, and Figure 2(b) compares different days.

Through the analysis of Figure 2, it can be seen that the outputs of different arrays are different, but the deviations are small. The array output varies significantly between different days, and the output of PV arrays shows strong volatil-

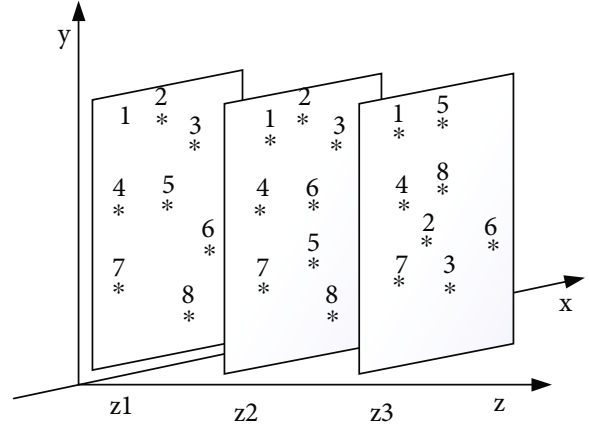

Figure 3: Schematic diagram of the three-dimensional data.

ity. Therefore, the deviation data of the array output is difficult to be used for PV fault diagnosis directly. Extracting the fault characteristics from the output deviations of different arrays and time series can be an effective way for the fault diagnosis. And this paper focuses on the extraction of the deviation characteristics of PV output and its application in array fault diagnosis. 


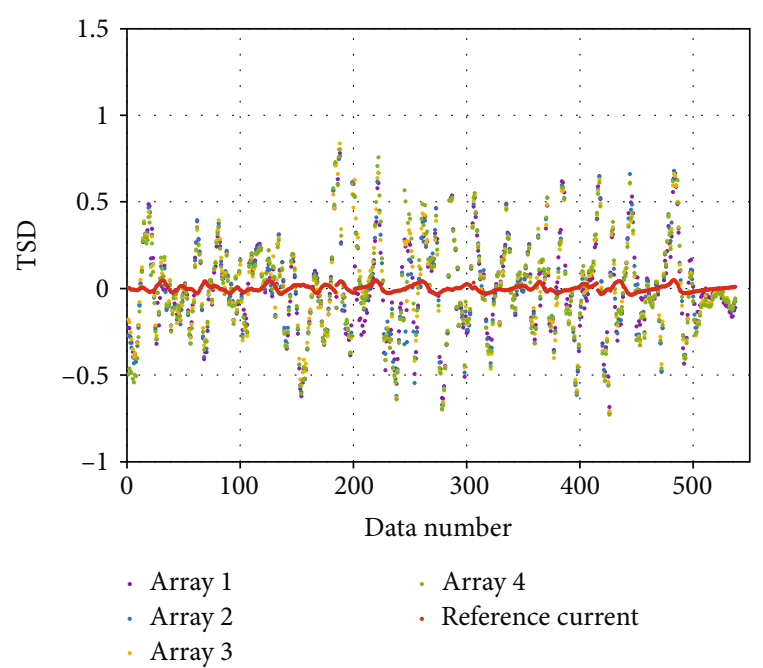

(a)

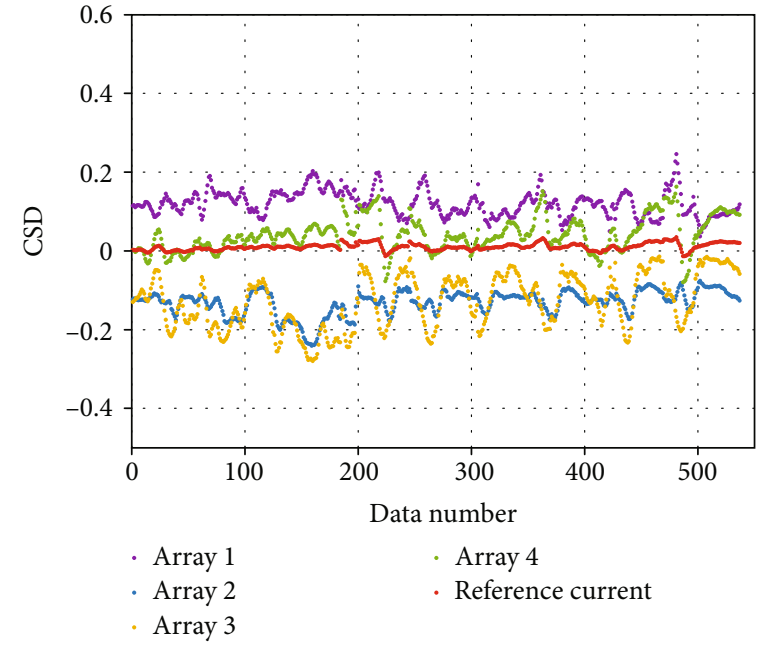

(b)

FIGURE 4: The TSD and CSD distributions of the array current. (a) The distribution of TSD. (b) The distribution of CSD.

\section{Description of the Deviation Distribution of the PV Array Output}

3.1. The Deviation Function. As shown in Figure 3, the threedimensional (3D) data are composed of multiple sets of twodimensional (2D) data. The 2D data are essentially the crosssectional data of different time series. The 3D data sets can show the differences between different samples and the changes in the sample evolution process.

The output deviation between different arrays and different time series can be used to construct 3D data that can show the output deviation characteristics of $\mathrm{PV}$ arrays. Suppose there are $N$ data samples, $x_{1}, x_{2}, \cdots, x_{N}$, and each data sample consists of a time series deviation component (TSD) and a cross-sectional deviation component (CSD).

TSD and CSD describe the deviation between different time series and the deviation of arrays of the array current, respectively. Therefore, the two components can be used to describe the PV output deviation.

The $k^{\text {th }}$ data sample, $x_{k}$, is represented as $x_{k}=\left[x_{k}(t) \|\right.$ $\left.x_{k}(s)\right]$, where the TSD equation is

$$
\left\{\begin{array}{l}
D_{T}\left(x_{T}, x_{S}\right)=I_{T, S}-I_{\mathrm{pre}, T, S} \\
I_{\mathrm{pre}, T, S}=a \bullet G+b
\end{array} .\right.
$$

$D_{T}\left(x_{T}, x_{S}\right)$ is the TSD of the array $S$ at time $T ; I_{T, S}$ is the measured current of the array $S$ at time $T ; I_{\text {pre, } T, S}$ is the reference current of the array $S$ at time $T$; the reference current is calculated from the curve equation obtained by fitting historical data; $a$ and $b$ are coefficients obtained by fitting the historical data; $G$ is the solar irradiation intensity at time $T$.

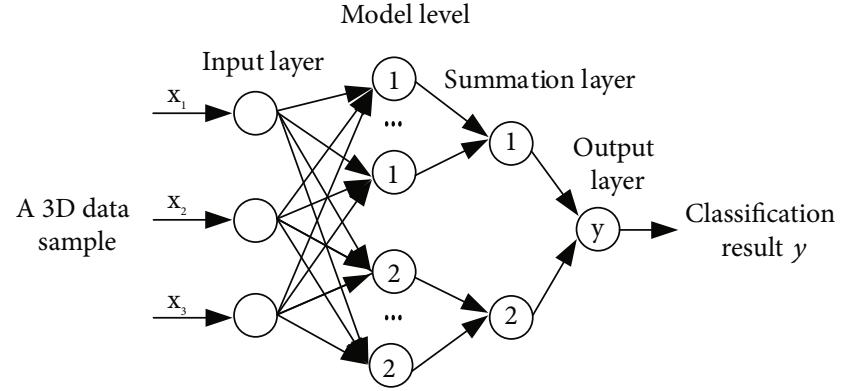

FIgURE 5: Structure diagram of a PNN.

The CSD function is

$$
\left\{\begin{array}{l}
D_{C}\left(x_{T}, x_{S}\right)=I_{T, S}-\bar{I}_{T}, \\
\bar{I}_{T}=\frac{\sum_{S=1}^{n} I_{T, S}}{n},
\end{array}\right.
$$

where $D_{C}\left(x_{T}, x_{S}\right)$ is the CSD of array $S$ at time $T ; \bar{I}_{T}$ is the reference current of the combiner box at time $T$, and $n$ is the number of arrays in the combiner box.

3.2. TSD and CSD Distributions of the Array Current. Figure 4 shows the variation of the deviation component of four arrays under normal conditions for 7 days. The data selected here are daytime data with 72 samples every day from 7 am to $7 \mathrm{pm}$, and the four arrays are selected from the same combiner box. The reference current here is calculated by Equation (1). Figure 4(a) shows the distribution of the TSD, and Figure 4(b) shows the distribution of the CSD. It can be seen from Figure 4 that the TSD of the fault-free array fluctuates with time, and the fluctuations are almost the same for different arrays. The CSD of PV arrays basically fluctuates in the range of -0.2 to 0.2 , with differences between the different arrays. The TSD and CSD 


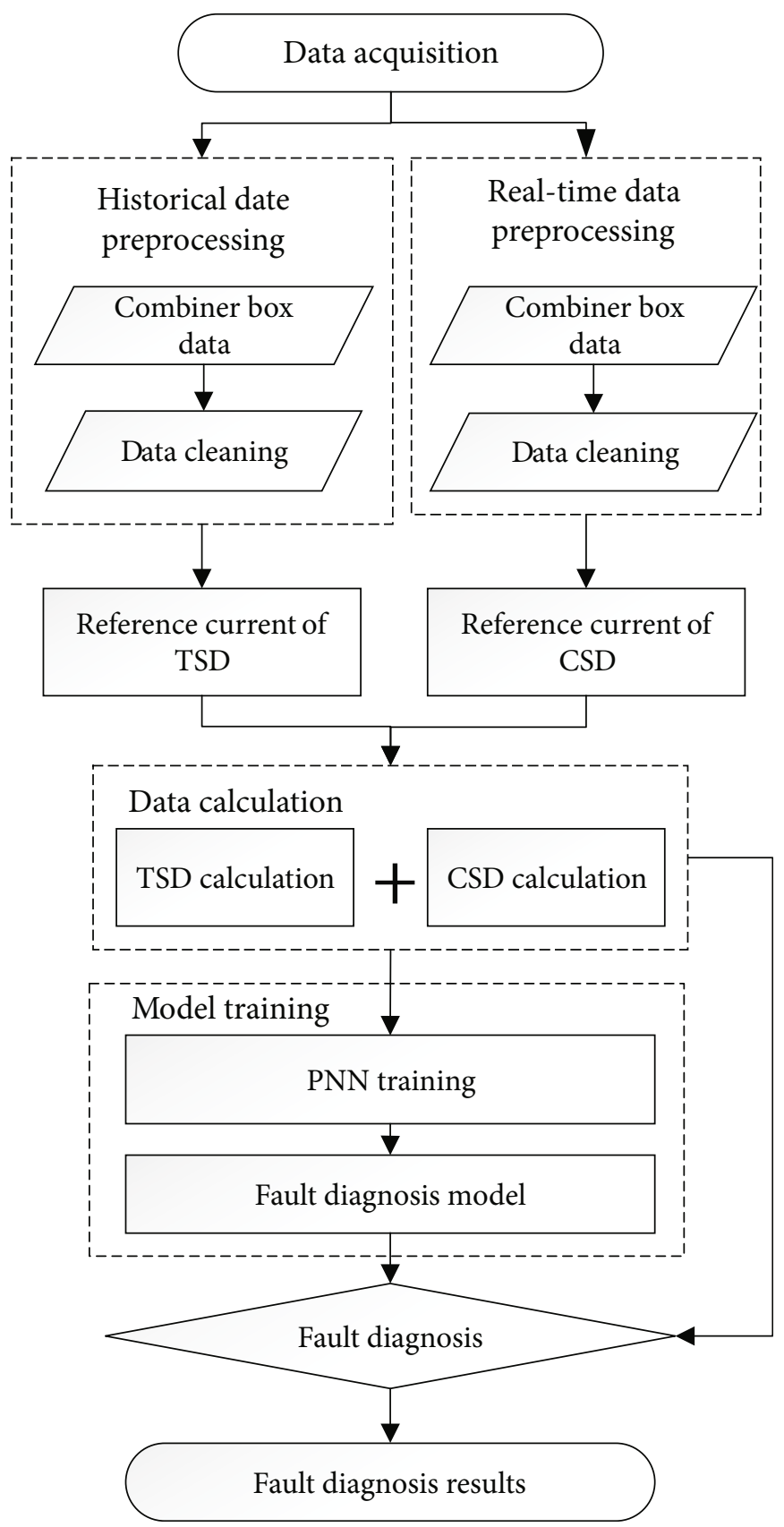

FIGURE 6: Flow chart of the proposed fault diagnosis method.

fluctuations of the reference current are very small compared with those of the actual array.

Through the analysis of Figure 4, the following conclusions can be drawn. Under normal operating conditions, the two indicators fluctuate within a certain range; TSD can effectively reflect the output deviation of different time series and CSD can effectively reflect the output deviation of different arrays. Therefore, these two indicators can be used for fault diagnosis of PV arrays.

\section{The Fault Diagnosis Method}

4.1. The Probabilistic Neural Network (PNN). The probabilistic neural network is a feedforward neural network developed from a radial basis function network. Based on the radial basis function neural network, the PNN integrates density function estimation and the Bayesian decision theory, and it is suitable for pattern classification [33]. Moreover, the PNN has the advantages of a simple network learning process, fast learning speed, accurate classification, high error and noise tolerance, and strong classification ability. Using the strong nonlinear classification ability of the PNN model, the failure mode of the sample space is mapped into fault space, and then it can build a fault diagnosis network system with strong structure of fault tolerance and self-adapted ability to improve the accuracy of diagnosis [34]. Based on the kernel estimation of probability density function of the PNN network, each training sample determines a sample of 
TABLE 1: Experimental platform.

\begin{tabular}{|c|c|c|}
\hline Item & Photos & Descriptions \\
\hline PV array & & $\begin{array}{c}\text { Model number: ZJN300 } \\
\text { Optimum operating voltage } V_{\mathrm{m}}: 36.5 \mathrm{~V} \\
\text { Optimum operating current } I_{\mathrm{m}}: 8.22 \mathrm{~A} \\
\text { Maximum power } p_{\mathrm{m}}: 300 \mathrm{kWp} \\
\text { Compensation coefficient: } 0.04 \% / \mathrm{K}\end{array}$ \\
\hline Combiner box & $\Delta$ & $\begin{array}{l}\text { Model number: PVS-16 M } \\
\text { Maximum number of input arrays: } 16 \\
\text { Rated output current: } 200 \mathrm{~A} \\
\text { Maximum input voltage: } 1100 \mathrm{~V}\end{array}$ \\
\hline Weather station & $:$ & $\begin{array}{l}\text { Model number: PYQX-02 } \\
\text { Collect ambient temperature } T \text {, horizontal irradiance } G \text {, } \\
\text { and other meteorological parameters of the PV power plant. }\end{array}$ \\
\hline Data collection system & & $\begin{array}{l}\text { Collect the current of the PV array, the voltage of the } \\
\text { combiner box, and the data collected by the } \\
\text { weather station, sampling interval is } 10 \text { minutes. }\end{array}$ \\
\hline
\end{tabular}

TABLE 2: Settings of different failure.

\begin{tabular}{lcc}
\hline Type of failure & Setting of failure & Remarks \\
\hline Normal & - & - \\
Aging & A series of $8 \Omega$ resistors in a PV array & - \\
Shading & Using an opaque cardboard to block a part of the & Blocking the half of \\
Open circuit & PV module in an array & 4 modules in an array \\
\hline
\end{tabular}

neurons, neuron weights directly from the input sample values. And the expansion of the PNN neural network is good, the learning process of network is simple, and to increase or decrease the number of pattern classes does not need a long training and learning time [35]. PNN has been widely used in the field of fault diagnosis [33-36], and it is suitable for fault diagnosis of PV array [27]. PNN has been chosen over other algorithms for fault detection and classification for a number of reasons. (1) The output of PV systems depends on environmental conditions. The PNN training system can develop its own decision boundaries based on the sampled data. (2) Simple classifiers such as fuzzy Cmeans clustering and $\mathrm{K}$-means clustering are likely to be stuck in a local optimum rather than reaching the global optimum. The intelligent PNN method, which uses heuristic method, is able to more efficiently reach the global optimum. (3) Output power from the PV array may drastically vary when there are momentary shadings due to clouds, rain, etc. PNN has the advantage of being relatively insensitive to these outliers unlike other simple classifiers and multilayer perceptron neural networks [27].
Therefore, this paper uses PNN as the tool for classification. The PNN is generally divided into four layers: the input layer, mode layer, sum layer, and output layer. (1) The input layer is responsible for transferring the feature vectors to the network and transferring the data to the hidden layer. The number of neurons in this layer is equal to the length of the input vector. (2) The mode layer connects with the input layer to calculate the matching degree between the input feature vector and each mode in the training set. The number of neurons in the mode layer is equal to the number of input sample vectors. (3) The summation layer obtains the estimated probability density function of the failure mode according to the probability accumulation results of a certain class. The number of neurons in this layer is equal to the number of sample categories. (4) The function of the output layer is to select a neuron with the maximum probability density from the estimated probability density of each fault mode as the output of the whole system.

The input and mode layers are connected by the Gaussian (Equation (4)), which is used to set the matching degree between each neuron in the mode layer and each neuron in 


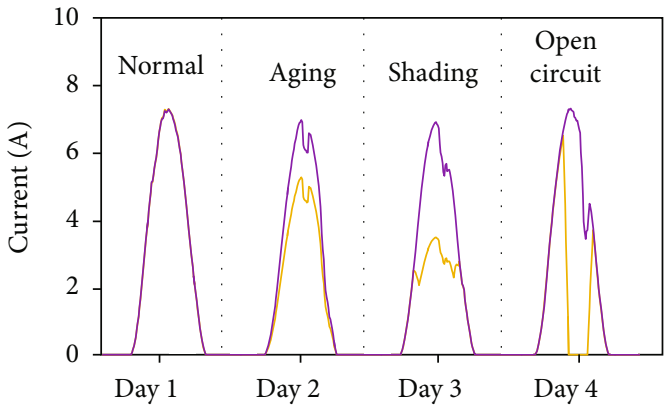

(a)

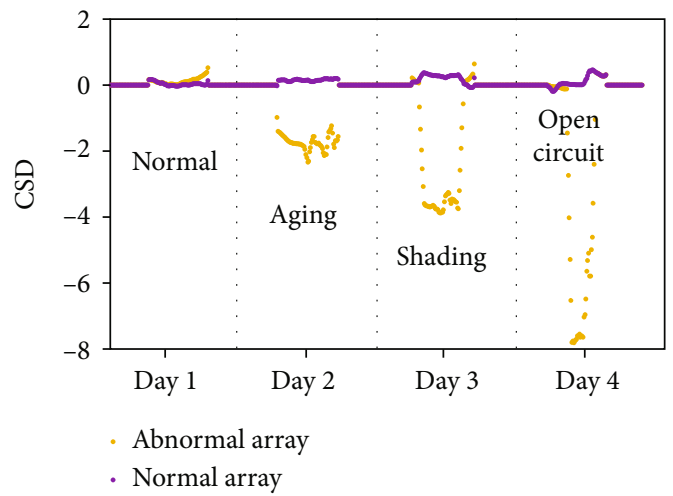

(c)

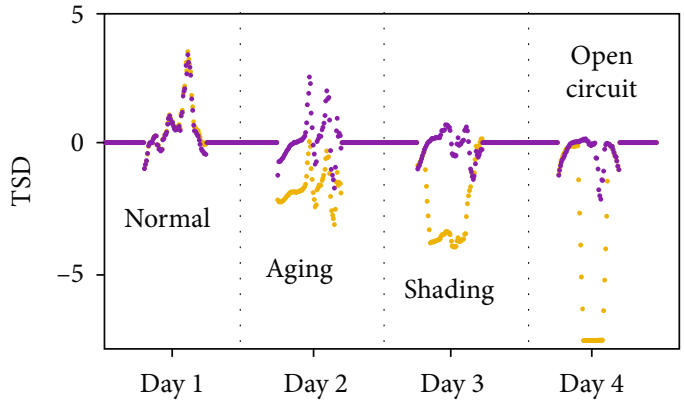

(b)

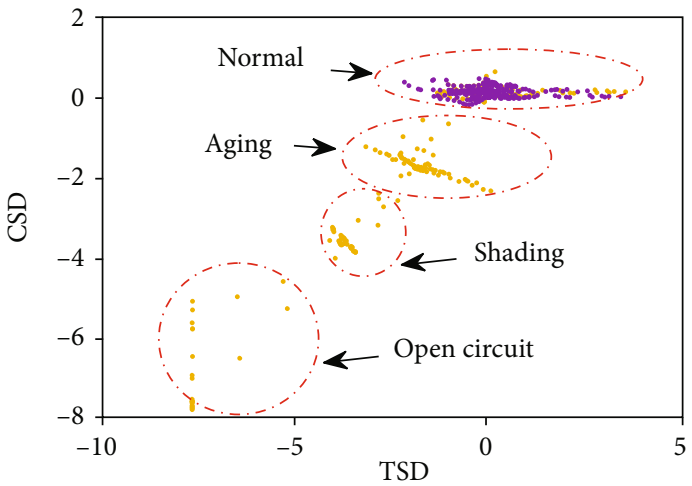

(d)

FIGURE 7: Output and corresponding TSD/CSD of the array current under different faults. (a) The deviation between the experimental array and normal array. (b) The TSD distribution under different faults. (c) The CSD distribution under different faults. (d) The deviation distribution under different faults.
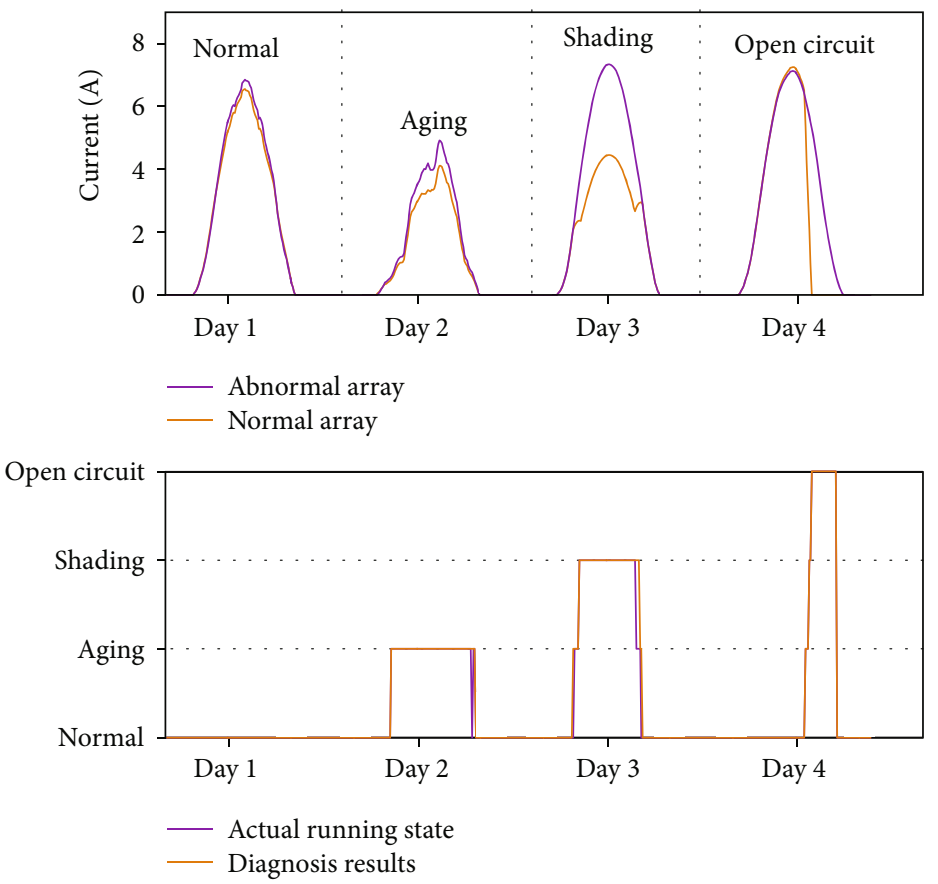

Figure 8: Fault diagnosis result. 
the input layer. By summing the matching degree of each class and taking the average, we can get the category of the input samples.

$$
y_{g}(x, \sigma)=\frac{1}{l_{g}^{m / 2} \sigma^{m}} \sum_{i=1}^{l_{g}} \exp \left(-\sum_{j=1}^{m} \frac{\left(x_{i, j}^{(g)}-x\right)^{2}}{2 \sigma^{2}}\right) .
$$

$y_{g}(x, \sigma)$ is the classification result of input vector $x$ under smoothing parameters $\sigma$; $l_{g}$ is the number of $g$ classes; $m$ is the sample dimension; $\sigma$ represents the smoothness parameters, which are generally between 0 and $1 ; x_{i, j}$ is the $j^{\text {th }}$ data sample of the $i^{\text {th }}$ neuron in class $g$.

Suppose there is a recognition task for two types of samples, and there is a variable number of samples for each type, and each sample has a $3 \mathrm{D}$ feature. Then, the network structure diagram can be drawn as in Figure 5.

4.2. Fault Diagnosis Method. The operation state of each array is basically the same under normal operation. When a fault occurs, the output deviation distribution of the fault array will be different from that of the normal array. Figure 6 presents the flow chart of the fault detection method proposed in this paper.

(1) Data Preprocessing. The historical and real-time data of the PV power plants are preprocessed. Data of night time is removed and only data with irradiance greater than $0 \mathrm{~W} / \mathrm{m}^{2}$ is used

(2) The Calculation of Deviation Component. The reference current of TSD is calculated using the historical data of irradiance and array current. The reference current of TSD under different irradiances is calculated according to Equation (2). The reference current of CSD is calculated using the real-time data. The reference current of CSD is calculated by using the current of each array in the same combiner box. After the reference current is obtained, based on the deviation function, the deviation component of each array can be calculated according to the difference between the actual value and the reference current of array current

(3) Training of the PNN Model. The array deviation component is taken as the input, and the array operation state is used as an output to train the PNN algorithm

(4) Fault Diagnosis. The real-time deviation component of each PV array is used as the input, and the trained PNN model is applied to diagnose the PV array

\section{Verification and Analysis}

5.1. Verification Data and Its TSD and CSD Distributions. Table 1 shows the configuration of the PV power plant. The following faults are analyzed in the paper: open circuit fault, abnormal aging, and shading. To illustrate the deviation characteristics of the array under different fault conditions
TABLE 3: Fault diagnosis accuracy statistics.

\begin{tabular}{lccc}
\hline $\begin{array}{l}\text { Type of } \\
\text { failure }\end{array}$ & $\begin{array}{c}\text { Sample } \\
\text { number }\end{array}$ & $\begin{array}{c}\text { Misjudged sample } \\
\text { number }\end{array}$ & $\begin{array}{c}\text { Accuracy } \\
\text { rate }\end{array}$ \\
\hline Normal & 65 & 0 & $100 \%$ \\
Aging & 66 & 1 & $98.5 \%$ \\
Shading & 64 & 1 & $98.4 \%$ \\
Open circuit & 66 & 2 & $97.7 \%$ \\
\hline
\end{tabular}

and verify the accuracy of the proposed method, a PV array (array A9 in combiner box M28 M366, contains 16 modules) is selected in the PV power plant for experimental verification. Different faults (normal, abnormal aging, shading, and open circuit) were set in array A9 for 20 days. The setting methods of each fault are described in Table 2. The 20-day experiment was divided into 5 groups, each group containing 4 days. The data of 4 groups are used for algorithm training, and the other data are used for method verification.

Figure 7 shows the deviation characteristics of the PV array output under different fault conditions. Figure 7(a) shows the comparison between the experimental array and normal array. The first day is fault free, and abnormal aging faults are set by series connection resistance in the experimental array on the second day; shading faults are set on the third day; and open circuit faults are set on the fourth day. Figure $7(\mathrm{~d})$ shows the distribution of the array output deviation under different faults. Figures 7 (b) and 7(c) show the TSD and CSD distributions of the normal array and fault array, respectively.

As shown in Figure 7, the distributions of the CSD and TSD of the PV array are obviously different under different fault conditions, which indicates that fault diagnosis of PV arrays based on the deviation characteristics is feasible.

5.2. Verification. The PNN model is trained using data collected every 10 minutes for 16 days. The 16 days were divided into four groups, and each group containing 4 days with different operating conditions of PV array. The radial basis function distribution density of the PNN is set to 0.5 . The results of the statistical analysis showed that the training accuracy of this model reached 0.9921 . The training accuracy of the PNN algorithm is high, so, the PNN model can effectively classify faults through CSD and TSD.

The performance of the proposed method is analyzed using 4 days experimental data. The data is collected from 7 am to 6 pm every 10 minutes and the setting conditions of faults are shown in Table 2. Figure 8 shows the fault diagnosis results for these 4 days. As shown in Table 3, the accuracy of the fault diagnosis is over $97 \%$. Therefore, the proposed method can detect different faults effectively.

5.3. Comparison. In order to demonstrate the superiority of the PNN algorithm, ANN (artificial neural network) and GRNN (generalized regression neural network) are selected for the comparison about the training speed and the training 


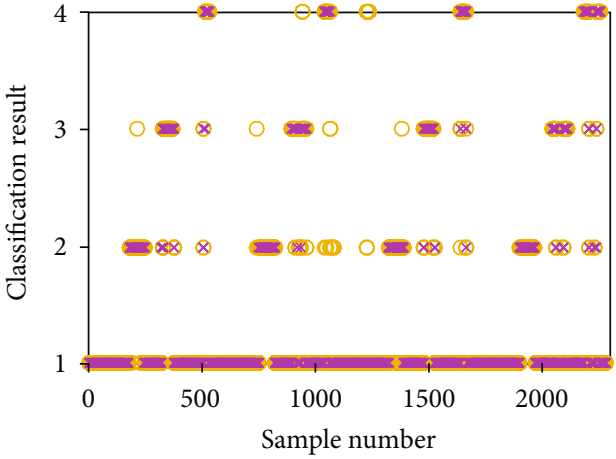

Predicted value

$\times$ Actual value

(a)

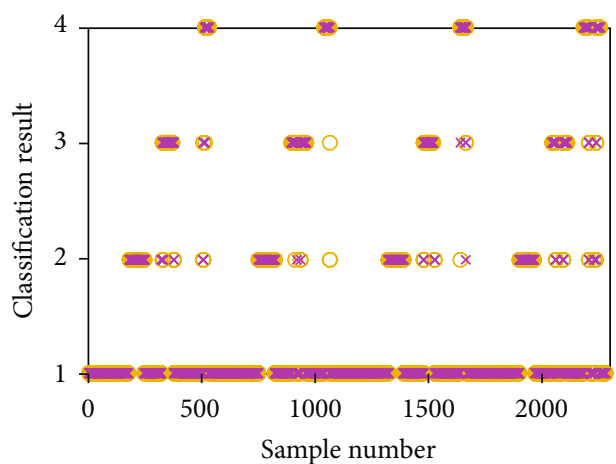

- Predicted value

$\times$ Actual value

(c)

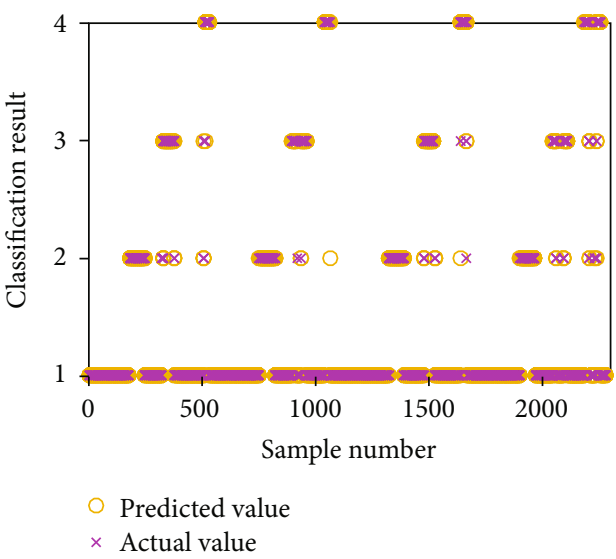

(e)

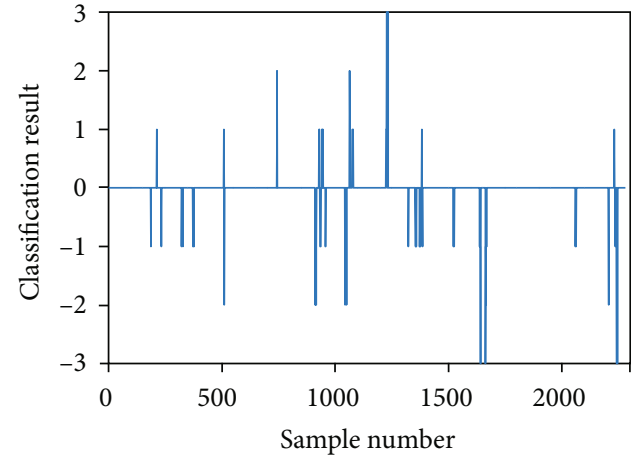

(b)

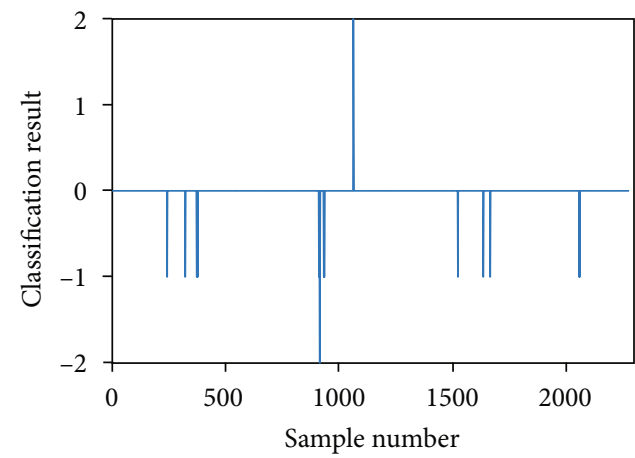

(d)

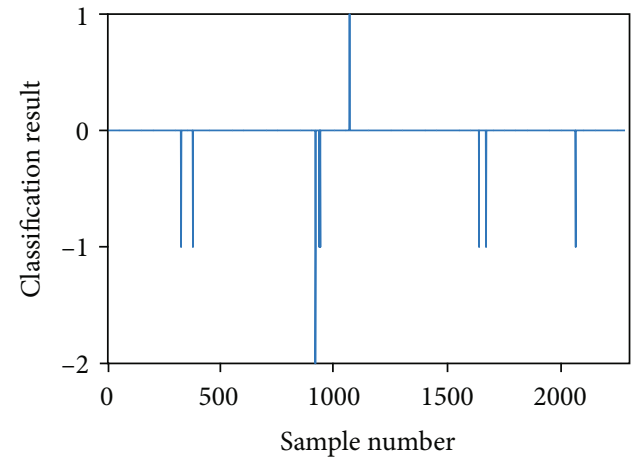

(f)

FIgURE 9: Training result comparison of different algorithms. (a) ANN training result. (b) ANN training error. (c) PNN training result. (d) PNN training error. (e) GRNN training result. (f) GRNN training error.

accuracy. The training results of each algorithm are shown in Figure 9.

It can be seen from Figure 9 and Table 4 that the training accuracy of these methods is above $95 \%$. But the training results of ANN are the worst, while the training accuracy of PNN and GRNN is above $99 \%$. Compared with PNN, GRNN has a significantly longer training time, so the PNN shows the best performance.

\section{Conclusion}

This paper studies the deviation characteristics of the PV array output and quantifies the deviation by the proposed function. Through the quantitative analysis of PV output deviation, it can be known that the deviation can be effectively used to identify the PV array fault. The PV array fault diagnosis method combining PNN algorithm with deviation 
TABle 4: Performance comparison of different algorithms.

\begin{tabular}{|c|c|c|c|}
\hline Type of algorithm & Parameter setting & Training time (s) & Training accuracy $(\%)$ \\
\hline ANN (artificial neural network) & $\begin{array}{c}\text { Network structure: }[200,4] \\
\text { Neuron activation function: }\{\text { 'logsig' 'purelin'\} } \\
\text { The target accuracy: } 0.001 \\
\text { Learning efficiency: } 0.01\end{array}$ & 0.456 & 0.9627 \\
\hline PNN (probabilistic neural network) & $\begin{array}{l}\text { Radial basis function distribution } \\
\text { density: spread }=0.5\end{array}$ & 0.360 & 0.9921 \\
\hline GRNN (generalized regression neural network) & $\begin{array}{l}\text { Radial basis function distribution } \\
\text { density: spread }=0.5\end{array}$ & 2.018 & 0.9947 \\
\hline
\end{tabular}

function is proposed. Finally, the method was verified in an actual PV power plant. The main findings and the shortcomings of the paper are summarized as follows:

(1) In the large-scale PV power plant, due to the parallel structure of the PV array, the actual available data is the PV array current. Therefore, based on the monitoring status of PV power plant, this paper proposes an effective method for fault diagnosis of PV arrays

(2) The deviation function realizes the quantification of the PV output deviation, effectively describes the output deviation of PV array between different time series and different arrays, and extracts the deviation characteristics of the PV output under different operating conditions

(3) The fault diagnosis of the PV array is carried out by combining the PNN algorithm with the PV array output deviation function. The proposed method is simple and effective, and is applicable for fault diagnosis of PV power plants

(4) The configuration and structure of different power plants are different, and the output characteristics of PV arrays are also different. So, the proposed method needs to be optimized for its applications in different PV power plants

\section{Data Availability}

The [Research Data IN EXCEL] data used to support the findings of this study are available from the corresponding author upon request.

\section{Conflicts of Interest}

The authors declare that there is no conflict of interest regarding the publication of this paper.

\section{Acknowledgments}

The authors thank the support of State Grid Henan Electric Power Company Research Institute to the laboratory activities. This research was funded by the Research Funds from State Grid Corporation of China "SGHADK00PJJS1800072."

\section{References}

[1] A. Triki-Lahiani, A. Bennani-Ben Abdelghani, and I. SlamaBelkhodja, "Fault detection and monitoring systems for photovoltaic installations: a review," Renewable and Sustainable Energy Reviews, vol. 82, no. 3, pp. 2680-2692, 2018.

[2] E. Kabir, P. Kumar, S. Kumar, A. A. Adelodun, and K.-H. Kim, "Solar energy: potential and future prospects," Renewable and Sustainable Energy Reviews, vol. 82, no. 1, pp. 894-900, 2018.

[3] D. S. Pillai and N. Rajasekar, "A comprehensive review on protection challenges and fault diagnosis in PV systems," Renewable and Sustainable Energy Reviews, vol. 91, no. 8, pp. 18-40, 2018.

[4] A. Mellit, G. M. Tina, and S. A. Kalogirou, "Fault detection and diagnosis methods for photovoltaic systems: a review," Renewable and Sustainable Energy Reviews, vol. 91, no. 8, pp. 1-17, 2018.

[5] S. R. Madeti and S. N. Singh, "A comprehensive study on different types of faults and detection techniques for solar photovoltaic system," Solar Energy, vol. 158, no. 12, pp. 161-185, 2017.

[6] Z. A. Jaffery, A. K. Dubey, Irshad, and A. Haque, "Scheme for predictive fault diagnosis in photo-voltaic modules using thermal imaging," Infrared Physics \& Technology, vol. 83, no. 7, pp. 182-187, 2017.

[7] P. Guerriero and S. Daliento, "Automatic edge identification for accurate analysis of thermographic images of solar panels," in 2017 6th International Conference on Clean Electrical Power (ICCEP), Santa Margherita Ligure, Italy, June 2017.

[8] S. R. Madeti and S. N. Singh, "Online modular level fault detection algorithm for grid-tied and off-grid PV systems," Solar Energy, vol. 157, no. 11, pp. 349-364, 2017.

[9] Y. Hu, J. Zhang, W. Cao et al., "Online two-section PV array fault diagnosis with optimized voltage sensor locations," IEEE Transactions on Industrial Electronics, vol. 62, no. 11, pp. 7237-7246, 2015.

[10] A. Livera, M. Theristis, G. Makrides, and G. E. Georghiou, "Recent advances in failure diagnosis techniques based on performance data analysis for grid-connected photovoltaic systems," Renewable Energy, vol. 133, no. 4, pp. 126-143, 2019.

[11] W. Chine, A. Mellit, V. Lughi, A. Malek, G. Sulligoi, and A. Massi Pavan, "A novel fault diagnosis technique for photovoltaic systems based on artificial neural networks," Renewable Energy, vol. 90, no. 5, pp. 501-512, 2016.

[12] F. Harrou, Y. Sun, B. Taghezouit, A. Saidi, and M.-E. Hamlati, "Reliable fault detection and diagnosis of photovoltaic systems based on statistical monitoring approaches," Renewable Energy, vol. 116, no. 2, pp. 22-37, 2018. 
[13] H. Yang, R. Cheng, C. Lv, and J. Li, "Fault diagnosis model based on parameter identification of photovoltaic module," Computer Measurement \& Control, vol. 26, no. 3, pp. 35-38, 2018.

[14] Y. Chaibi, M. Malvoni, A. Chouder, M. Boussetta, and M. Salhi, "Simple and efficient approach to detect and diagnose electrical faults and partial shading in photovoltaic systems," Energy Conversion and Management, vol. 196, no. 9, pp. 330-343, 2019.

[15] G. Fu, X. Li, and X. Gao, "Analytical method and application of current discrete rate of $\mathrm{PV}$ power station based on junction box string," Power System and Clean Energy, vol. 30, no. 11, pp. 109-113, 2014.

[16] Q. Liu, Y. Zhao, Y. Zhang, D. Kang, Q. Lv, and L. Shang, "Hierarchical context-aware anomaly diagnosis in large-scale PV systems using SCADA data," in 2017 IEEE 15th International Conference on Industrial Informatics (INDIN), Emden, Germany, July 2017.

[17] M. Dhimish and V. Holmes, "Fault detection algorithm for grid-connected photovoltaic plants," Solar Energy, vol. 137, no. 11, pp. 236-245, 2016.

[18] M. Mansouri, M. Hajji, M. Trabelsi et al., "An effective statistical fault detection technique for grid connected photovoltaic systems based on an improved generalized likelihood ratio test," Energy, vol. 159, no. 9, pp. 842-856, 2018.

[19] E. Garoudja, F. Harrou, Y. Sun, K. Kara, A. Chouder, and S. Silvestre, "Statistical fault detection in photovoltaic systems," Solar Energy, vol. 150, no. 7, pp. 485-499, 2017.

[20] C. Ventura and G. M. Tina, "Development of Models for Online Diagnostic and Energy Assessment Analysis of PV Power Plants: The Study Case of 1 MW Sicilian PV Plant," Energy Procedia, vol. 83, no. 11, pp. 248-257, 2015.

[21] L. C. Chen, P. J. Lin, J. Zhang et al., "Fault diagnosis and classification for photovoltaic arrays based on principal component analysis and support vector machine," IOP Conference Series: Earth and Environmental Science, vol. 188, 2018.

[22] Z. Chen, L. Wu, S. Cheng, P. Lin, Y. Wu, and W. Lin, "Intelligent fault diagnosis of photovoltaic arrays based on optimized kernel extreme learning machine and I-V characteristics," Applied Energy, vol. 204, no. 10, pp. 912-931, 2017.

[23] A. Livera, M. Theristis, G. Makrides, and G. E. Georghiou, "On-line failure diagnosis of grid-connected photovoltaic systems based on fuzzy logic," in 2018 IEEE 12th International Conference on Compatibility, Power Electronics and Power Engineering (CPE-POWERENG 2018), Doha, Qatar, April 2018.

[24] Z. Chen, F. Han, L. Wu et al., "Random forest based intelligent fault diagnosis for PV arrays using array voltage and string currents," Energy Conversion and Management, vol. 178, no. 12, pp. 250-264, 2018.

[25] Z. Chen, Y. Chen, L. Wu, S. Cheng, and P. Lin, “Deep residual network based fault detection and diagnosis of photovoltaic arrays using current-voltage curves and ambient conditions," Energy Conversion And Management, vol. 198, no. 111793, p. 111793, 2019.

[26] Z. Chen, Y. Chen, L. Wu, S. Cheng, P. Lin, and L. You, "Accurate modeling of photovoltaic modules using a 1-D deep residual network based on I-V characteristics," Energy Conversion and Management, vol. 186, no. 4, pp. 168-187, 2019.

[27] M. N. Akram and S. Lotfifard, "Modeling and health monitoring of DC side of photovoltaic array," IEEE Transactions on Sustainable Energy, vol. 6, no. 4, pp. 1245-1253, 2015.
[28] Y. Hu, J. Zhang, P. Li, D. Yu, and L. Jiang, "Non-uniform aged modules reconfiguration for large-scale PV array," IEEE Transactions on Device and Materials Reliability, vol. 17, no. 3, pp. 560-569, 2017.

[29] Y. Hu, J. Zhang, J. Wu, W. Cao, G. Y. Tian, and J. L. Kirtley, "Efficiency improvement of non-uniformly-aged PV arrays," IEEE Transactions on Power Electronics, vol. 32, no. 2, pp. 1124-1137, 2017.

[30] H. Zhu, H. Wang, D. Kang et al., "Study of joint temporalspatial distribution of array output for large-scale photovoltaic plant and its fault diagnosis application," Solar Energy, vol. 181, no. 3, pp. 137-147, 2019.

[31] H. Wang, J. Zhao, Q. Sun, and H. Zhu, "Probability modeling for PV array output interval and its application in fault diagnosis," Energy, vol. 189, no. 116248, p. 116248, 2019.

[32] H. Zhu, W. Lian, L. Lu et al., "Online modelling and calculation for operating temperature of silicon-based PV modules based on BP-ANN," International Journal of Photoenergy, vol. 2017, Article ID 6759295, 13 pages, 2017.

[33] G. L. Zuo, S. D. Lai, and Y. Cheng, "Study on the fault diagnosis of gear pump based on PNN neural network," Advanced Materials Research, vol. 1044-1045, no. 10, pp. 873-876, 2014.

[34] S. Li, X. Li, and W. Wang, "Fault diagnosis of transformer based on probabilistic neural network," in 2011 Fourth International Conference on Intelligent Computation Technology and Automation, Shenzhen, Guangdong, China, March 2011.

[35] F. Zhang, Y. Wang, and F. Chen, "Classification of building electrical system faults based on probabilistic neural networks," 2016 Chinese Control and Decision Conference (CCDC), 2016, Yinchuan, China, May 2016, 2016.

[36] R. Ouhibi, S. Bouslama, and K. Laabidi, "Faults classification of asynchronous machine based on the probabilistic neural network (PNN)," in International Conference on Control Engineering \& Information Technology, Hammamet, Tunisia, December 2016. 